\title{
Deployable Helical Antennas for CubeSats
}

\author{
Gina M. Olson ${ }^{1}$ and Sergio Pellegrino ${ }^{2}$ \\ California Institute of Technology, Pasadena, CA, 91125 \\ Jeremy Banik ${ }^{3}$
}

Air Force Research Laboratory, Space Vehicles Directorate, Kirtland Air Force Base, New Mexico, 87117

and

Joseph Costantine ${ }^{4}$

California State University - Fullerton, Fullerton, CA, 92834

\begin{abstract}
This paper explores the behavior of a self-deploying helical pantograph antenna for CubeSats. The helical pantograph concept is described along with concepts for attachment to the satellite bus. Finite element folding simulations of a pantograph consisting of eight helices are presented and compared to compaction force experiments done on a prototype antenna. Reflection coefficient tests are also presented, demonstrating the operating frequency range of the prototype antenna. The helical pantograph is shown to be a promising alternative to current small satellite antenna solutions.
\end{abstract}

\section{Introduction}

S mall satellites, such as CubeSats, are faster and cheaper to develop than traditional satellites and have a higher risk tolerance. As part of a push for greater capabilities in small satellites, solar sails, space weather monitors and space telescopes have been recently developed for the CubeSat platform [1-3]. However, the comparatively slow bit rates between these satellites and ground stations limits downlink intensive applications, such as Earth imaging or relaying communications [4]. A high gain, wideband deployable antenna suitable for small satellites could increase their downlink speed and enable capabilities found on much larger satellites.

The predominant antenna choice for CubeSats has been one or more monopole or dipole antennas [5]. Dipole antennas are omnidirectional and two or more can be arranged to mimic circular polarization, a desired quality that limits a satellite's required pointing control. Furthermore, dipoles are straightforward to design and deploy, and designs specific to CubeSats are available commercially. Examples of CubeSats that have flown with dipole antennas include Tokyo Institute of Technology's Cute 1.7+ APD II and California Polytechnic's CP1 [6,7].

Although omnidirectional, dipoles are low gain, naturally linearly polarized, inefficient and normally narrowband [5]. Helical antennas are wider band antennas and are naturally circularly polarized. A half wavelength dipole antenna is limited to a gain of $2.15 \mathrm{dBi}$, while helical antennas can have gains in excess of $8 \mathrm{dBi}$ [8]. Helical antennas may also have more than one helix connected through a balun to further increase their gain.

The potential bit rate of an antenna depends on its gain and bandwidth, and is limited by that bandwidth and the availability of power on the satellite bus. Consider an antenna transmitting in the presence of Gaussian noise; the channel capacity can then be estimated with the Shannon Hartley theorem, which gives

$$
\text { bps }=B \log _{2}\left(1+\frac{S}{N}\right)
$$

where bps is the bit rate in bits/second, $B$ is the bandwidth of the antenna and $S / N$ is the signal to noise ratio of the transmission. A typical fractional bandwidth for a thin dipole is 3\% [9]. Assuming a transmitting power to noise

${ }^{1}$ Graduate Student, Graduate Aerospace Laboratories, 1200 E. California Blvd, MC 205-45, AIAA Student Member,golson@caltech.edu

2 Joyce and Kent Kresa Professor of Aeronautics and Professor of Civil Engineering, Graduate Aerospace Laboratories, 1200 E. California Blvd, MC 301-46, AIAA Fellow, sergiop@ caltech.edu

${ }^{3}$ Research Mechanical Engineer, 3550 Aberdeen Ave SE, AIAA Senior Member

4 Assistant Professor, Electrical Engineering Department, $800 \mathrm{~N}$ State College Blvd, E-100A jcostantine@fullerton.edu 
power ratio of two, a dipole with a central operating frequency of $365 \mathrm{MHz}$ and a gain of $2.15 \mathrm{dBi}$ would have a channel capacity of $15.9 \mathrm{Mbps}$. The quadrifilar helix antenna discussed in this paper has a bandwidth of $27.5 \mathrm{MHz}$ and a gain of $8 \mathrm{dBi}$, which gives a channel capacity of $71.8 \mathrm{Mbps}$. If the bit rate is only limited by the power available, the increase is proportional to the gain increase between antennas; in this case the quadrifilar helix bit rate would be approximately four times the dipole bit rate.

Packaging a high gain UHF antenna into a CubeSat is a challenging problem because the size of an antenna is inversely proportional to its frequency and the dimensions of a CubeSat impose a tight constraint, with a $1 \mathrm{U}$ measuring $10 \mathrm{~cm}$ on each side and a $3 \mathrm{U}$ CubeSat measuring only $10 \mathrm{~cm} \times 10 \mathrm{~cm} \times 30 \mathrm{~cm}$. Compare these dimensions to a quadrifilar helix antenna receiving $365 \mathrm{MHz}$, which typically measures $12 \mathrm{~cm}$ in diameter and is $0.5 \mathrm{~m}$ tall.

In [10], four structural concepts suitable for packaging UHF antennas on small satellites with limited pointing control were proposed. The present paper focuses on further study of the helical pantograph structure from [10]. The helical pantograph is a new structural concept and hence our objective is to understand and explain its behavior. In addition to finite element folding simulations, we have made a physical model of a helical pantograph antenna on which we have conducted structural and electromagnetic tests.

Section II describes the helical pantograph concept, including two possibilities for attachment to the satellite bus. Section III presents finite element simulations of the compaction of the structure. Section IV describes a prototype antenna and the compaction force experiments that were used to verify the folding simulations, as well as a reflection coefficient test to verify the operating frequency range. Section $\mathrm{V}$ concludes the paper.

\section{Concept Description and Analytical Basis}

\section{A. Helical Pantograph Concept and Background}

The helical pantograph concept was inspired by the linear pantograph in [11], which is a mechanism capable of significant changes of length. A helical pantograph is formed by connecting together two opposite sense helices with the same diameter and pitch, by means of a revolute joint aligned with the radial direction, at every crossover point. Increasing the helix angle lengthens the structure, while decreasing the helix angle shortens the structure. Unlike the linear pantograph, this structure relies on elastic deformation in addition to relative rotation of the joints and so is not a mechanism. Hence, the structure has the potential to deploy by releasing its stored strain energy, rather than relying on deployment systems that provide external energy, such as cables or motors. A detailed description of this concept and its development are presented in [10].

Axial compaction of a helical pantograph increases its radius, but transverse compaction is also possible by pulling the structure towards its central axis, into a shape resembling a four-leaf clover. Figure 1 illustrates this twostage compaction process for a helical pantograph consisting of a right-handed helix (blue) and a left-handed helix (gold) connected by pivots at seven crossover points.

A helical pantograph consisting of only two helical rods is rather compliant, but a stiffer structure can be formed by increasing the number of helices and adding further connections at all additional crossover points. Commercially available helical antennas have one, two or four same-sense conducting helices. A helical antenna with four conducting helices is referred to as a quadrifilar helix antenna; example is shown in Figure 2. Due to the structural and electromagnetic benefits of quadrifilar helix antennas, this paper will focus on helical pantographs consisting of four conductors supported by four opposite sense helices, for a total of eight helices. However, this work is also applicable to structures with a different number of helices.

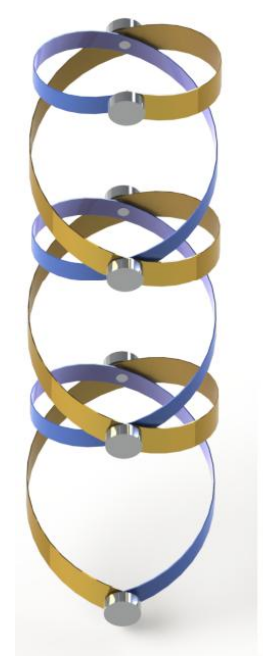

(a) Extended

Figure 1. Helical pantograph shown (a) fully deployed, (b) compacting axially and (c) transversely compacted.

2

American Institute of Aeronautics and Astronautics 
The eight helices have the same pitch $c$ and radius $r$. The parametric equation of the right-handed helical curves is

$$
\begin{gathered}
x=r \cos \left(\theta-\theta_{i}\right) \\
y=r \sin \left(\theta-\theta_{i}\right) \\
z=c \theta
\end{gathered}
$$

where the offset $\theta_{\mathrm{i}}$ is $0, \pi / 2, \pi$ and $3 \pi / 2$ radians. The parametric equation of the left-handed helices is

$$
\begin{gathered}
x=r \cos \left(\theta-\theta_{i}\right) \\
y=-r \sin \left(\theta-\theta_{i}\right)
\end{gathered}
$$

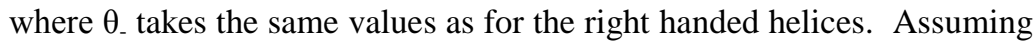
that all helices have cross section of height $a$ and width $b$, the radius $R_{S I}$ of the structure at the end of Stage I folding can be estimated by equating the arc-length of the original helix to a helix with a pitch equal to the cross-section height

$$
R_{S I}=\sqrt{r^{2}+c^{2}-\left(\frac{a}{2 \pi}\right)^{2}}
$$

The minimum stowed height $H$ of this structure can be estimated from

$$
H_{S I}=N\left(\frac{n}{2}+1\right) a
$$

where $N$ is the number of turns of each helix, $n$ is the number of helices (e.g. 2, 4, 8). In addition to the geometric analysis presented here, for any chosen set of cross section dimensions and material properties, a detailed analysis needs to be carried out to confirm that folding the structure does not cause damage or permanent deformation of the helices.

\section{B. Analytical Solutions for Helices Made From Straight Rods}

A helical pantograph may either be formed from initially straight or initially helical rods. All solutions presented in this section are also applicable to strips with an appropriate torsion constant $J$. If initially straight rods are used, some combination of boundary conditions (e.g. twisting moment, force, rotation) must be used to enforce a helical shape, while initially helical rods or strips are naturally in equilibrium in the helical configuration.

There is a standard analytical solution for the bending and twisting of straight rods into a helical shape, a version of this solution is presented in [12]. Even if initially helical rods are used, this solution provides useful insight into how a helical pantograph structure might behave, and the boundary conditions required to maintain a helical shape.

For the helical curve defined in Equation 2 with $\theta_{0}=0$, the tangent vector $\boldsymbol{t}$ is given by

$$
\begin{gathered}
t_{x}=-r \sin \theta \\
t_{y}=r \cos \theta \\
t_{z}=c
\end{gathered}
$$

while the binormal vector $\boldsymbol{b}$ is given by

$$
\begin{gathered}
b_{x}=r c \sin \theta \\
b_{y}=-r c \cos \theta \\
b_{z}=r^{2}
\end{gathered}
$$

and both are shown in Figure 3. The torsion of the helix is

$$
\tau=\frac{c}{r^{2}+c^{2}}
$$


The bending and twisting moments in the initially straight rod are then

$$
\boldsymbol{M}=E I \frac{(\cos \alpha)^{2}}{r} \boldsymbol{b}+G J \tau \boldsymbol{t}
$$

while the internal force, assuming no stretch in the helix, is

$$
\boldsymbol{T}=\frac{(\cos \alpha)^{2}}{r}\left(G J \tau-\frac{E I}{r} \cos \alpha \sin \alpha\right) \boldsymbol{b}
$$

and both are uniform along the entire rod. In the above equations, $\alpha$ is the helix angle, $E$ is the elastic modulus, $G$ is the shear modulus, $I$ is the vertical cross section second moment of area and $J$ is the torsion constant.

Consider how this solution applies to a helical pantograph formed by two helices made from identical rods, that have the same radius and pitch, but turn in an opposite sense. The end reactions, at $\mathrm{A}$ and $\mathrm{B}$, are defined in a common Cartesian coordinate system, shown in Figure 3. The binormal and tangent moments at $\mathrm{A}$ and $\mathrm{B}$ can be decomposed into $\mathrm{Z}$ and $\mathrm{Y}$-components. The $\mathrm{Z}$-components for the twisting and bending moments in the right- and lefthanded helices are equal and opposite, hence their resultants are zero, while the moments about $\mathrm{Y}$ will add.

For helices formed from straight rods there are several boundary condition combinations that can produce a solution in static equilibrium. One solution is to apply a force parallel to $\mathrm{Z}$ and a twisting moment about the tangent vector at $A$ and $B$ [13].

If initially helical rods are used, there will no reactions in the initial shape. If the helix is then deformed by compacting it, while allowing radial expansion, boundary conditions can be imposed such that in every configuration

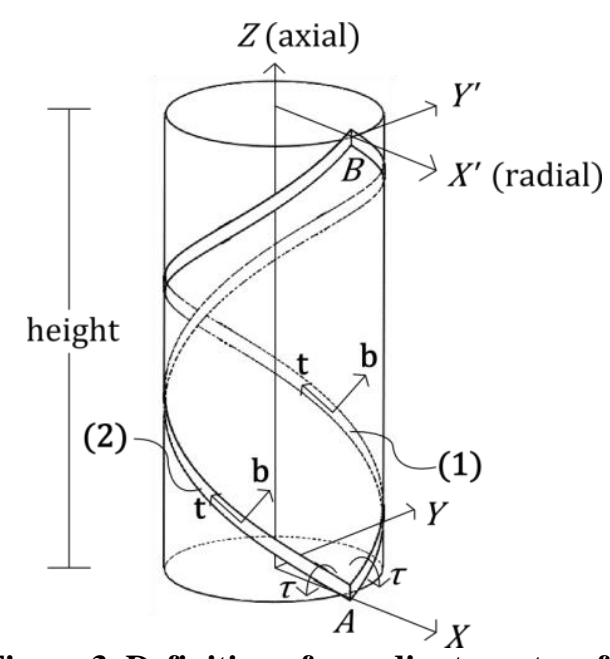

Figure 3. Definition of coordinate system for right-handed (1) and left-handed (2) helices.

the rods will still be helical. The solution above suggests that the application of a twisting and bending moment along with a compressive force at $\mathrm{A}$ and $\mathrm{B}$ would achieve this.

\section{Attachment to Satellite Bus}

The interface between the CubeSat and the structure must allow significant displacement of the attachment points, as the antenna expands in the transverse direction during Stage I folding and then contracts during Stage II folding (see Figure 1). The exact shape the helices take during transverse compaction depends on the packing constraints, helix dimensions and material. Two concepts have been identified, both suitable for the application discussed in this paper.

The first concept involves the three-dimensional expansion of a structure consisting of four identical links, and is shown in Figure 4.

\section{Deployed}

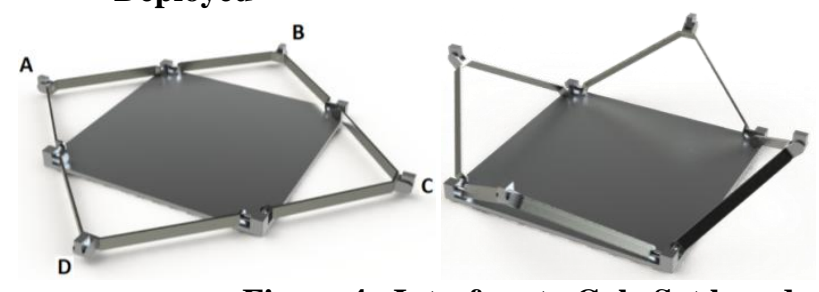

Folded

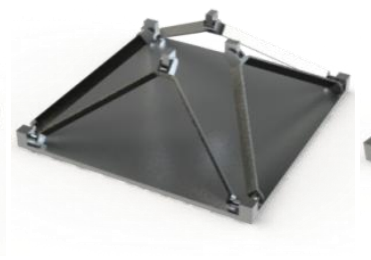

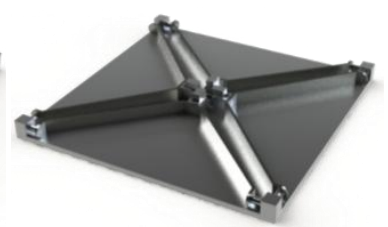

Figure 4. Interface to CubeSat based on three dimensional link concept.

The four links shown are attached to a square plate, representing a face of a CubeSat, via revolute joints. These joints allow each link to rotate about an axis parallel to a side of the square. At the tip of each of the link there is another revolute joint through which the link is connected to the helical pantograph. 
The maximum distance between the link tips in the deployed and folded configurations is achieved when the links rotate through $180^{\circ}$. Allowing for a clearance of $2 \mathrm{~mm}$, the largest antenna diameter that can be supported by this concept has a radius after axial compaction of $7.4 \mathrm{~cm}$. The links could be made longer to provide a larger extended diameter, but then they would not be able to fold completely flat, resulting in a taller package.

The second concept was derived from the dilator cell [14] shown in Figure 5. The dilator cell is a planar mechanism consisting of four identical right triangles of height $h$ and base length $s$, hinged at a base corner to a square of side length $l$. The triangles are connected by links, also of length $l$, parallel to the sides of the square. Denoting by $\lambda$ the side length of the square formed by the tips of the triangles, $\lambda$ decreases when $\theta$ varies from $135^{\circ}$ to $0^{\circ}$. The minimum and maximum values of $\lambda$ are [14]

$$
\begin{gathered}
\lambda_{\min }=\sqrt{2 h^{2}+l^{2}-2 h l} \\
\lambda_{\text {max }}=l+\sqrt{2} h
\end{gathered}
$$

where $l$ is the side length of the internal square and $h$ the height of the triangular link. The base length $s$ of the triangular link does not affect the minimum and maximum size, but $s$ must be smaller than $l$.

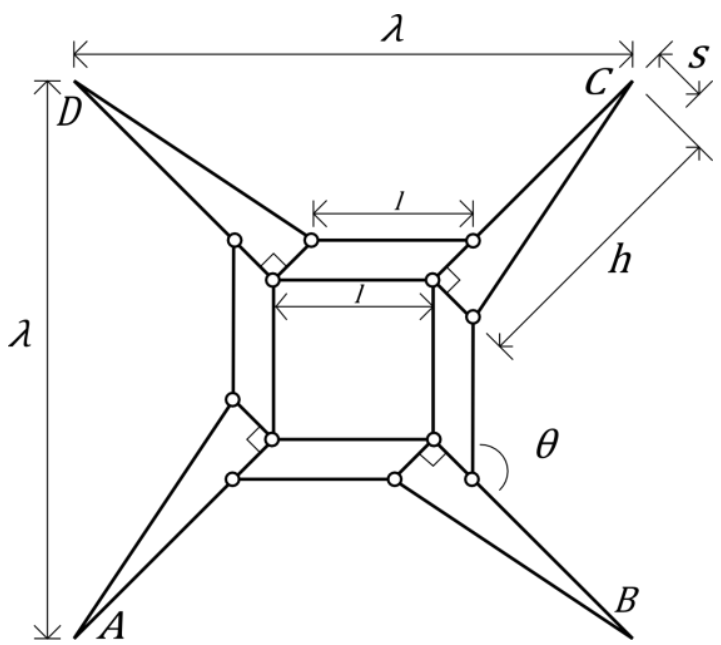

Figure 5. Interface to CubeSat based on dilator cell from [14].

Among these two concepts, the dilator cell is simpler and works entirely in a plane. It provides as much compaction as the prior concept and has a single degree of freedom.

\section{Numerical Simulations of Folding}

\section{A. Axial Folding}

A finite element simulation of Stage I folding of the helical pantograph was done in ABAQUS/Standard, with the helices modeled with shell elements. Nodes and elements were generated via a script which discretizes the helical curves and for each cross-section arranges a set of node points on the helix binormal. The number of nodes and elements was selected such that on each helix there would be a node at every cross over point, and the helices were pinned together at both ends, by imposing translational constraints and only allowing a relative rotation about the radial axis.

Initially, a simple pantograph consisting of two single-turn helices was considered to examine the interaction between the helices. Each helix had a cross section of $6 \mathrm{~mm} \times$ $0.240 \mathrm{~mm}$ and was assumed to be made of beryllium copper, which has an elastic modulus of $130 \mathrm{GPa}$ and Poisson's ratio of 0.3 . The initial helix radius was $6.06 \mathrm{~cm}$ and the pitch $37.1 \mathrm{~cm}$.

The results of these analyses are presented in terms of the coordinate system shown in Figure 3. The radial direction corresponds to the $X^{\prime}$ axis, while the transverse axis corresponds to the $Y^{\prime}$ axis. The pantograph height is the distance between the $X$ and $X^{\prime}$ axes, and a downward displacement boundary condition at the top of each helix was used to

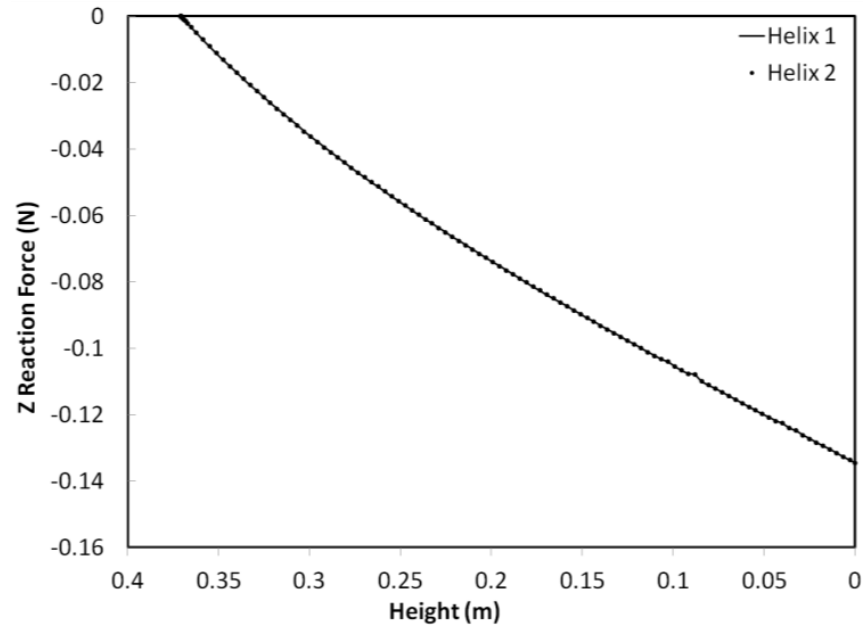

Figure 6. Z-reaction forces for two-helix pantograph. compact the structure.

Figure 6 shows plots of the Z-components of the reaction forces at the top of each helix as the pantograph is compressed. As suggested by the analytical solution, the forces on the two helices are equal and in the same sense; 
they increase monotonically and are noticeably nonlinear as the (tangent) stiffness of the pantograph decreases at greater degrees of compaction. Both the $\mathrm{X}^{\prime}$ - and $\mathrm{Y}^{\prime}$ - reaction components are smaller than the resolution of the analysis and may be considered to be zero.

The Y'- reaction moments are shown in Figure 7, while the Z-reaction moments are shown in Figure 8. The X'reaction moment is zero because $\mathrm{X}^{\prime}$ is the axis of the revolute joint. The $\mathrm{Y}^{\prime}$ - reaction moments on the two helices are equal and have the same sign, while the Z-reaction moments are equal and opposite.

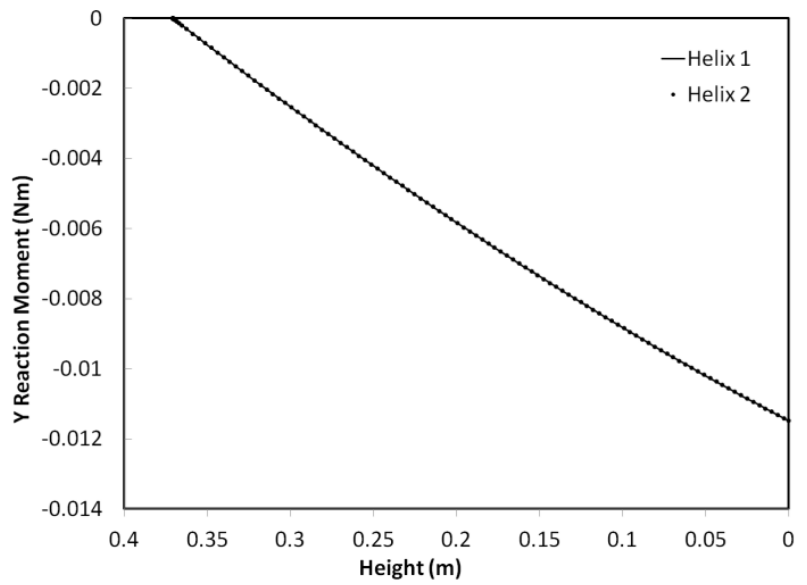

Figure 7. Y'-reaction moments for two-helix pantograph.

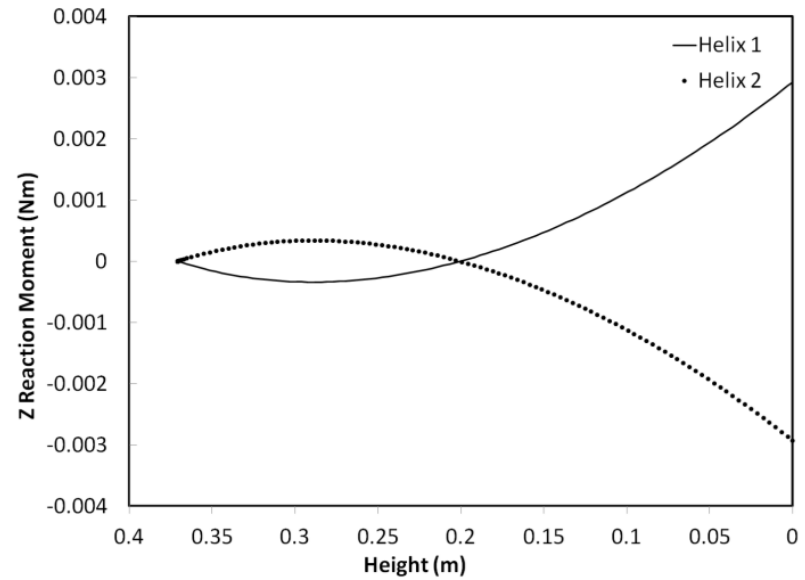

Figure 8. Z-reaction moments for two-helix pantograph.

Next, a simulation of the eight-helix structure matching the design dimensions of the prototype described in Section IV.A was analyzed. One set of helices was made of beryllium copper, with the geometry and properties provided above; the other set was made of unidirectional S-2 glass composite. The S-2 glass helices were assigned a cross section of $15.8 \mathrm{~mm} \times 0.2 \mathrm{~mm}$ and the material constants, determined from tensile and shear tests, were longitudinal modulus of $50 \mathrm{GPa}$ and Poisson's ratio of 0.2 . Four images from the simulation of axial compaction are shown in Figure 9.
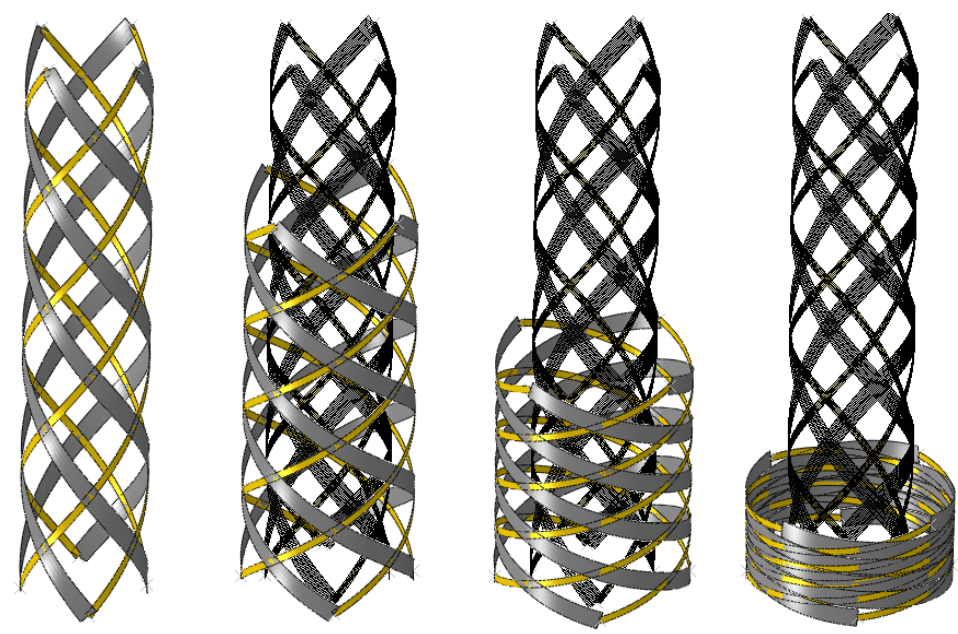

Figure 9. Images from FEA simulation of compaction of eight-helix pantograph. 
The axial reaction force for this structure is shown in Figure 10, along with the axial reaction forces for a S-2 glass helix and a beryllium copper helix. Figures 11 and 12 show plots of the reaction moments at one of the end joints. Because the cross sections of the two sets of helices are not matched, the moment cancellation seen in Section II.B does not occur in the present case.

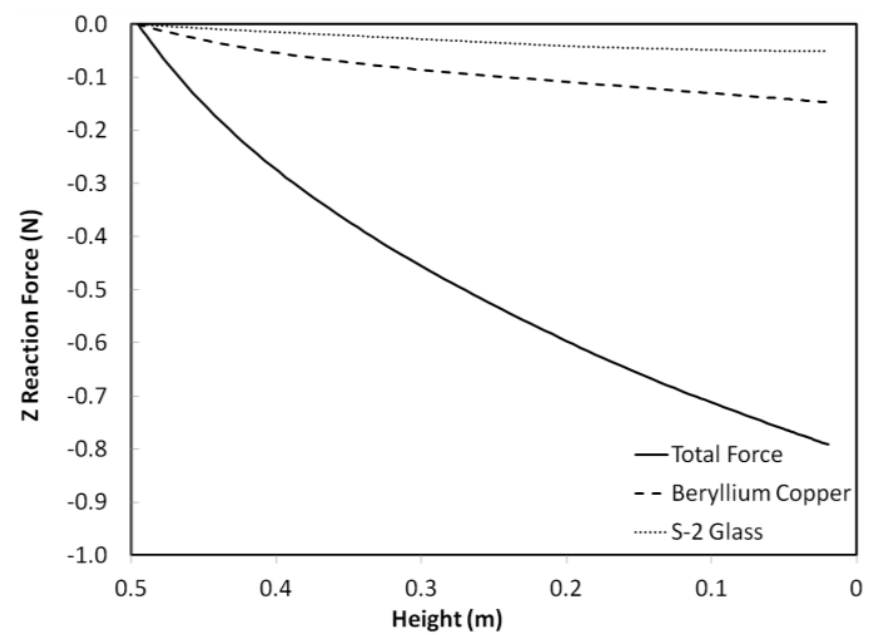

Figure 10. Z-reaction forces for eight-helix pantograph.

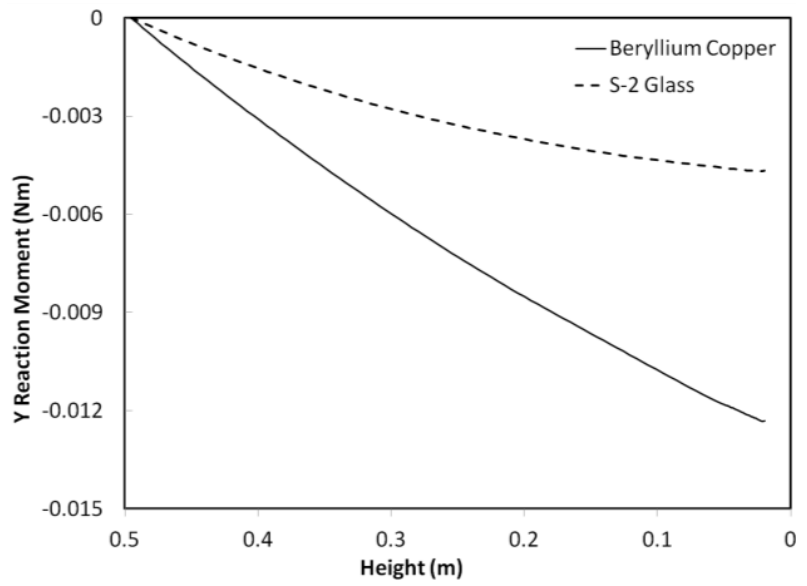

Figure 11. Eight-helix $Y^{\prime}$-reaction moment.

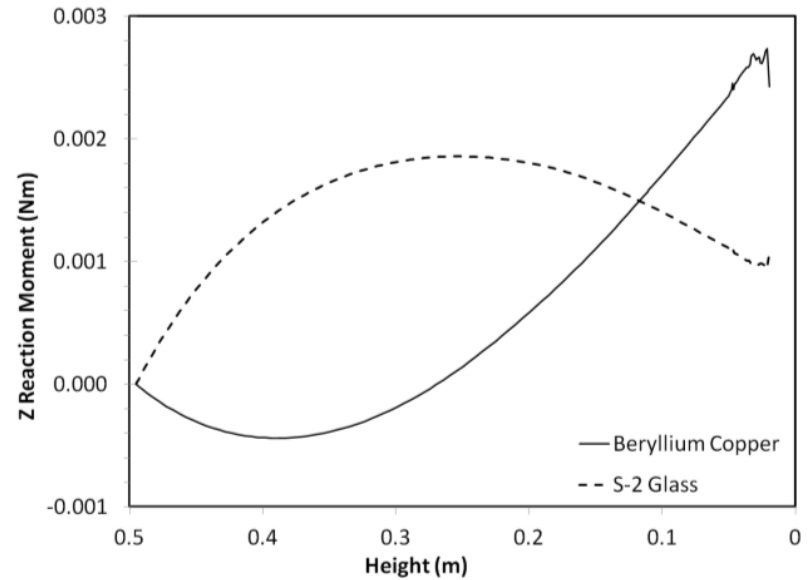

Figure 12. Eight-helix Z- reaction force.

\section{B. Transverse Folding}

The transverse folding scheme was studied with ABAQUS/Standard using a simple finite-element model of the structure consisting of a single circular ring beam. The compaction progress outlined in Section II.C was implemented, and the simulation was used to determine the compacted shape and resulting curvatures in the ring beam. First, two pairs of diametrically opposite points were displaced radially inward until self-contact was achieved, Figure 13(a-c). Second, four points at $45^{\circ}$ to the first four were displaced inward, Figure 13(d). Note that both Figure 13(c) and (d) demonstrate Step 2 compactions that reduce the transverse dimensions of the pantograph enough to fit inside a CubeSat. The interior four attachment points could be held in place with burn wire or a similar solution. 


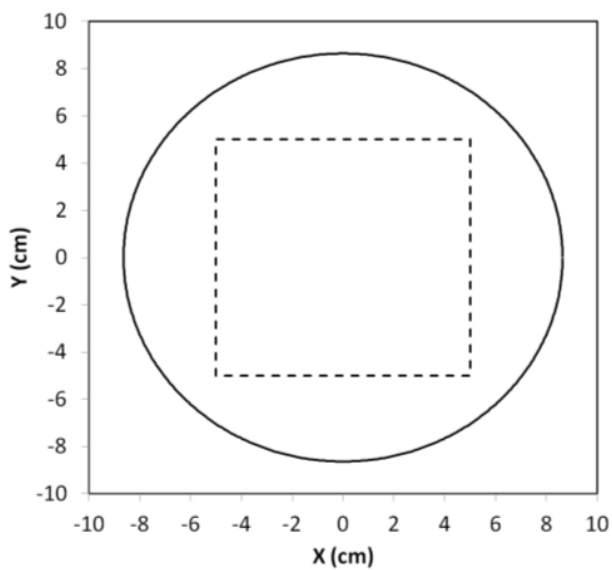

(a)

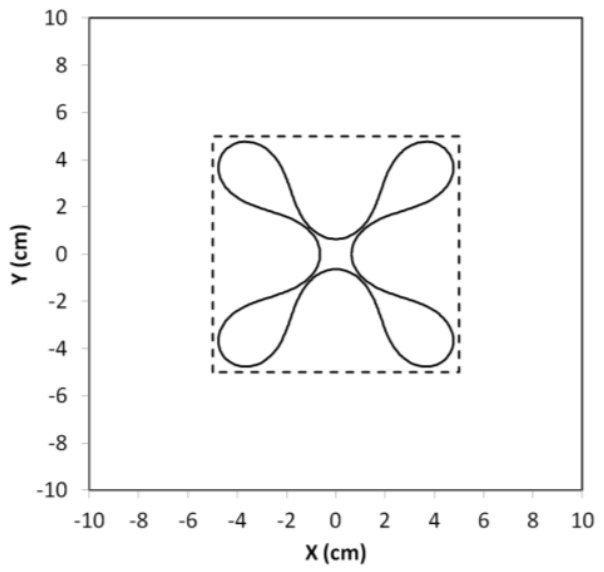

(b)

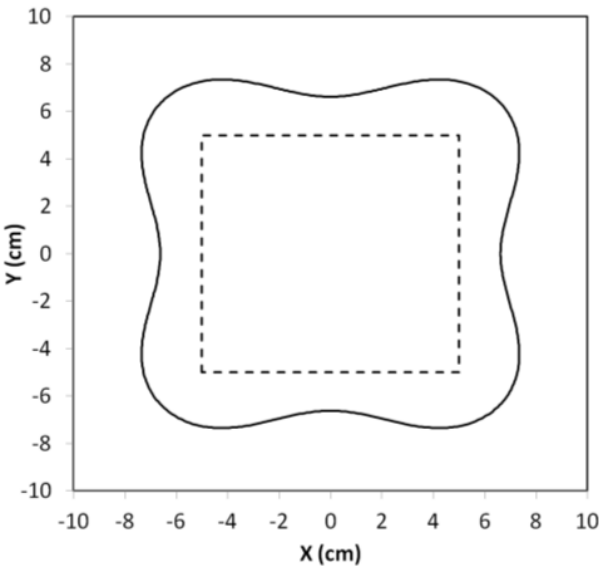

(c)

Figure 13. Transverse compaction process.

Also note that the configuration shown in Figure 13(d) could be further compacted by pulling in the $45^{\circ}$ points.

Figure 14 shows plots of the curvatures associated with the compacted configurations shown in (c) and (d), for increasing node number (the nodes are evenly spaced in the original configuration). Note that the maximum curvature remains substantially unchanged.

Using these results, the dimensions of a dilator cell that attaches to points A, B, C, D at the base of the helical pantograph can be calculated. After axial compaction, these points lie at $( \pm 6.19 \mathrm{~cm}, \pm 6.19 \mathrm{~cm})$ using the coordinate system identified in Figure 3, while after transverse compaction they are at $( \pm 4.44$ $\mathrm{cm}, \pm 4.44 \mathrm{~cm})$. There are several suitable sets of dimensions, but the solution implemented

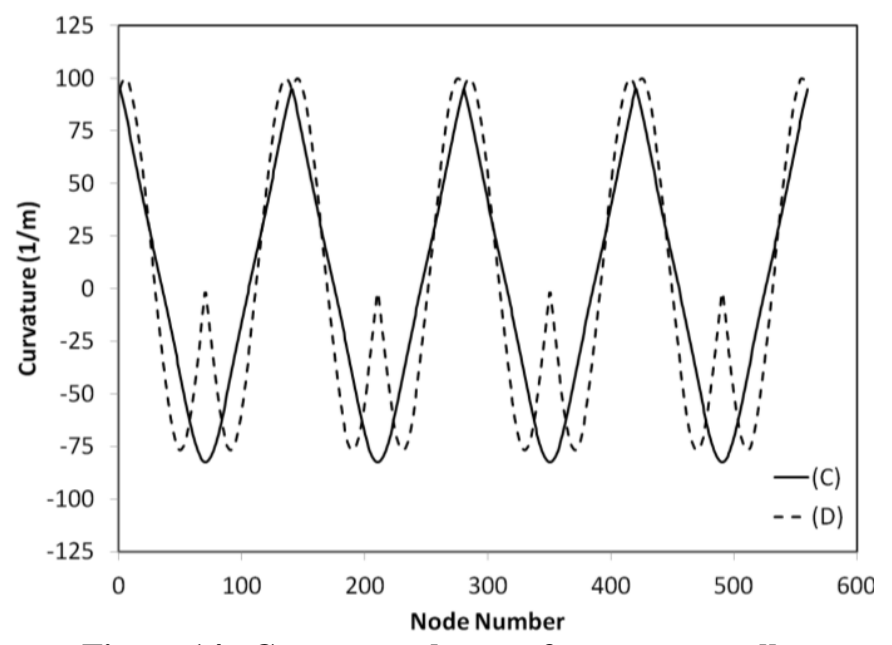

Figure 14. Curvature changes for transversally compacted configurations in Figure 13(c) and (d).

here is: $l=3.46 \mathrm{~cm}, h=6.5 \mathrm{~cm}$ and $s=1.34 \mathrm{~cm}$. Figure 15 shows models of the dilator mechanism in several states of compaction, as well as the dilator cell connected to a fully extended helical pantograph. 


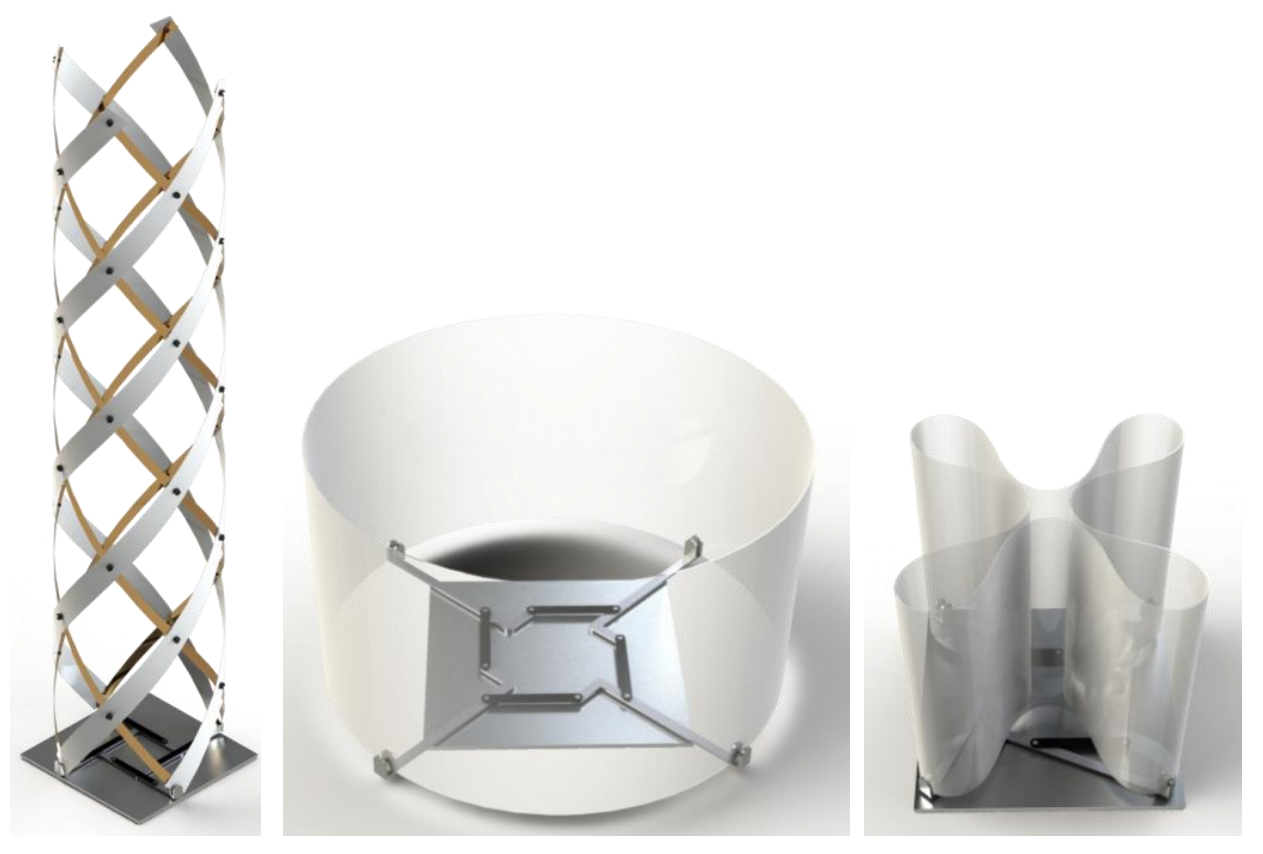

Figure 15. Dilator cell interface mechanism.

\section{Experiments}

\section{A. Prototype Construction}

A prototype of the eight-helix helical pantograph was built to demonstrate the deployment functionality and to measure the compaction force to compare to the finite element model. The designed dimensions of the prototype conductors came from an electromagnetic simulation of the antenna, which predicted it would operate on a frequency range centered around $365 \mathrm{MHz}$. The selected dimensions were: helix diameter of $12.12 \mathrm{~cm}$ and pitch of $37.1 \mathrm{~cm}$ with 1.125 turns and a conductor cross section of $6 \mathrm{~mm} \times 0.240 \mathrm{~mm}$. However, the manufactured helix diameter was measured at $10.2 \mathrm{~cm}$.

The conducting helices were made from Alloy 25 beryllium copper, heat treated to increase the yield stress and take a helical shape. The support helices were made from a unidirectional S-2 glass fiber toughened epoxy composite. The dimensions of the support helices were found by matching a combination of geometric and material parameters in an attempt to match torsion and axial stiffness. Specifically, the conditions

$$
\begin{aligned}
E_{c} A_{c} & =E_{s} A_{s} \\
J_{c} G_{c} & =J_{s} G_{s}
\end{aligned}
$$

were imposed, where the subscript $c$ means conductor and the subscript $s$ means support. The approximation for the torsion constant

$$
J=\left(\frac{a}{2}\right)\left(\frac{b}{2}\right)^{3}\left[\frac{16}{3}-3.36 \frac{b}{a}\left(1-\frac{b^{4}}{12 a^{4}}\right)\right]
$$

was used, where $a$ is the cross section height as before and $b$ is the cross section width [15]. This approximation is valid for rectangular cross sections with $a$ much greater than $b$. The cross-sectional dimensions of the support helices were obtained from Equations 13 and 14, where classical lamination theory had been used to estimate the composite material properties. The final selected dimensions for the support helices were $15.8 \mathrm{~mm} \times 0.2 \mathrm{~mm}$ $(0.625$ " $\mathrm{x} 0.008$ "). However, the actual measured dimensions of the manufactured helices were $15.7 \mathrm{~mm} \times 0.27 \mathrm{~mm}$ to $0.52 \mathrm{~mm}$. The measured width at the center of the cross section varied from $0.27 \mathrm{~mm}$ to $0.52 \mathrm{~mm}$. In addition, the mandrel used to cure the glass composite strips into a helical form left ridges on the cross section edges of parts of 
the helix; these ridges were $\sim 0.5 \mathrm{~mm}$ high $\times 0.25 \mathrm{~mm}$ wide on top of the helix width and were difficult to remove without cracking the helices.

The described design process is linear; the dimensions for the conductors were determined from a simulation of the antenna, and then the dimensions of the support helices were determined. This process was used for this prototype to simplify the approach, but is not the desired final design process as it doesn't allow for structural optimization.

After manufacturing, both the conductive and support helices were essentially strain free in the deployed configuration. The helices were attached with nonconductive screws and thin plastic washers were used to separate the helices at all connection points, to reduce friction. The finished prototype can be seen in various phases of compaction in Figure 16.
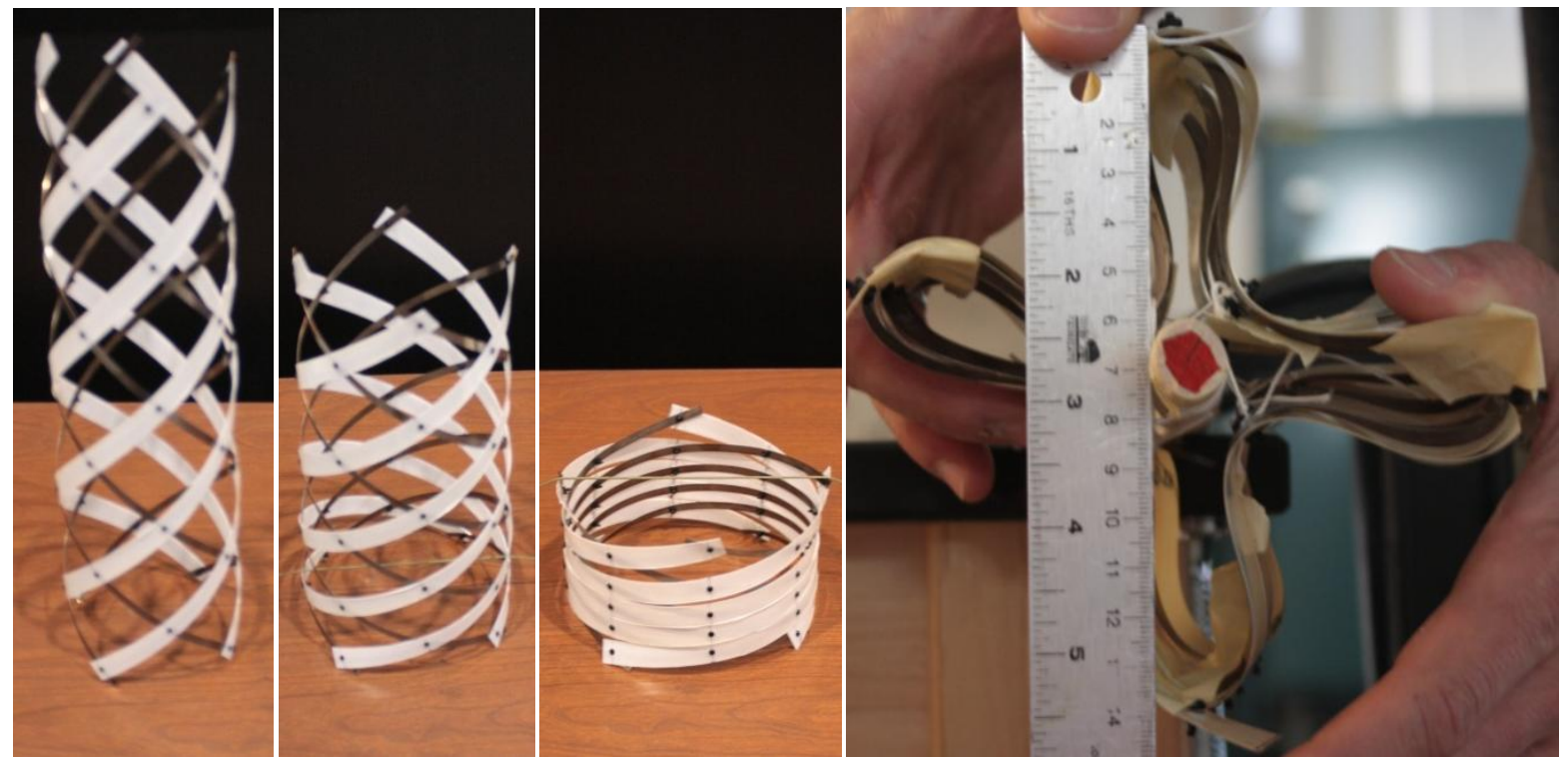

Figure 16. Folding of prototype antenna.

\section{B. Compaction Force Measurement}

A helical pantograph is difficult to test due to its large deformation during compaction and the difficulty of maintaining the appropriate boundary conditions during this process. As the structure compacts axially it also expands transversally, which makes the test even harder to design. While a direct compression test may seem an intuitive choice, designing a low friction compression test set-up that provides the correct boundary conditions on the helices, with minimal mass and friction, is difficult.

Instead of applying direct compression on the structure, the structure was tested in a hanging configuration and the axial force required to axially compact the structure (Stage I folding) was applied by means of symmetrically arranged cords looped over miniature pulleys attached to the structure itself. Specifically, four pulleys were attached to pairs of crossover points of the helical pantograph, two on each side, at an initial separation of $0.22 \mathrm{~m}$ in the initial, deployed configuration. Nylon cords were looped around the pulleys such that pulling on these cords shortens the loops and hence decreases the height of the prototype antenna. The cord loops were terminated on ultra-low friction linear slides (one at the top, one at the base) to allow radial expansion of the prototype as it is folded. This test set-up is shown in Figure 17.

Results from three load-unload cycles are shown in Figure 18.

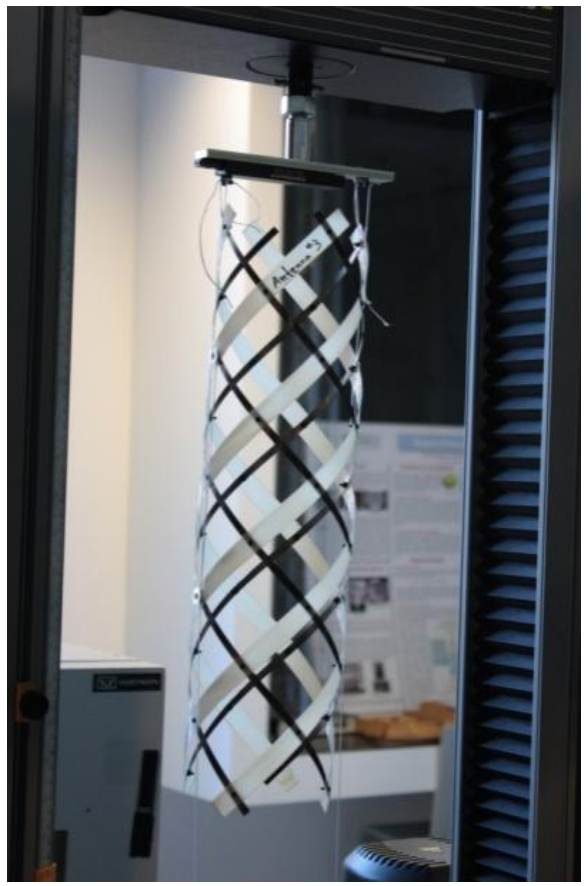

Figure 17. Stage 1 folding test set-up. 
The results are repeatable, but show a large difference between loading and unloading, due to friction between the pulleys and the prototype, which increases the load while the prototype is being compacted, and takes up part of the load while the prototype is extending. A demonstration of this explanation is provided in Figure 19, showing plots of measurements taken while loading the structure alternately at a rate of $0.5 \mathrm{~mm} / \mathrm{s}$ for periods of $40 \mathrm{~s}$ and a rate of $0.001 \mathrm{~mm} / \mathrm{s}$ for periods of 50 s. During the periods of slower loading rate the structure was tapped lightly (always in the same place) to release friction between the pulleys and the cords. Figure 19(a) shows the measured load vs. measurement time, while Figure 19(b) shows the same load measurements but plotted against total antenna height.

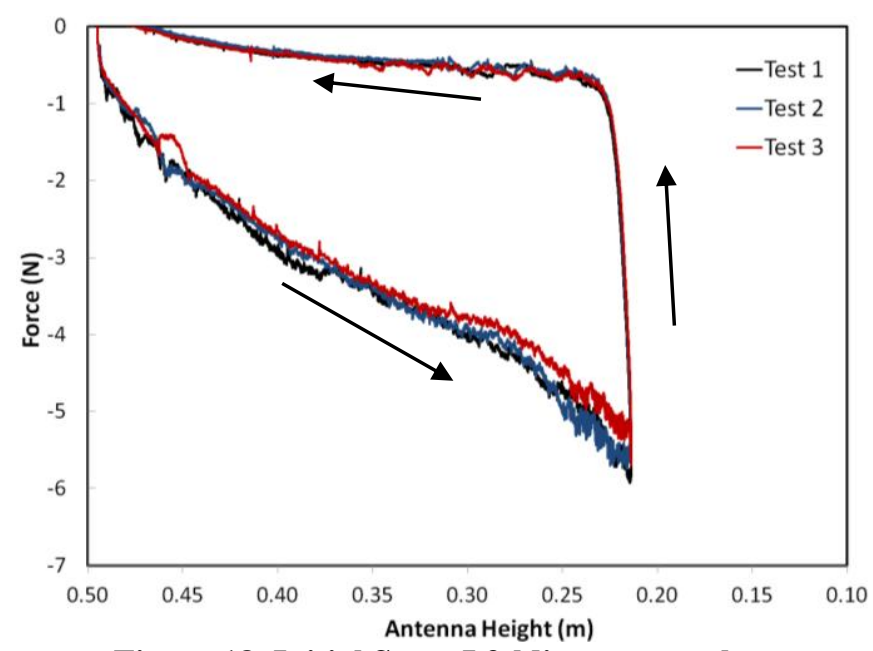

Figure 18. Initial Stage I folding test results.
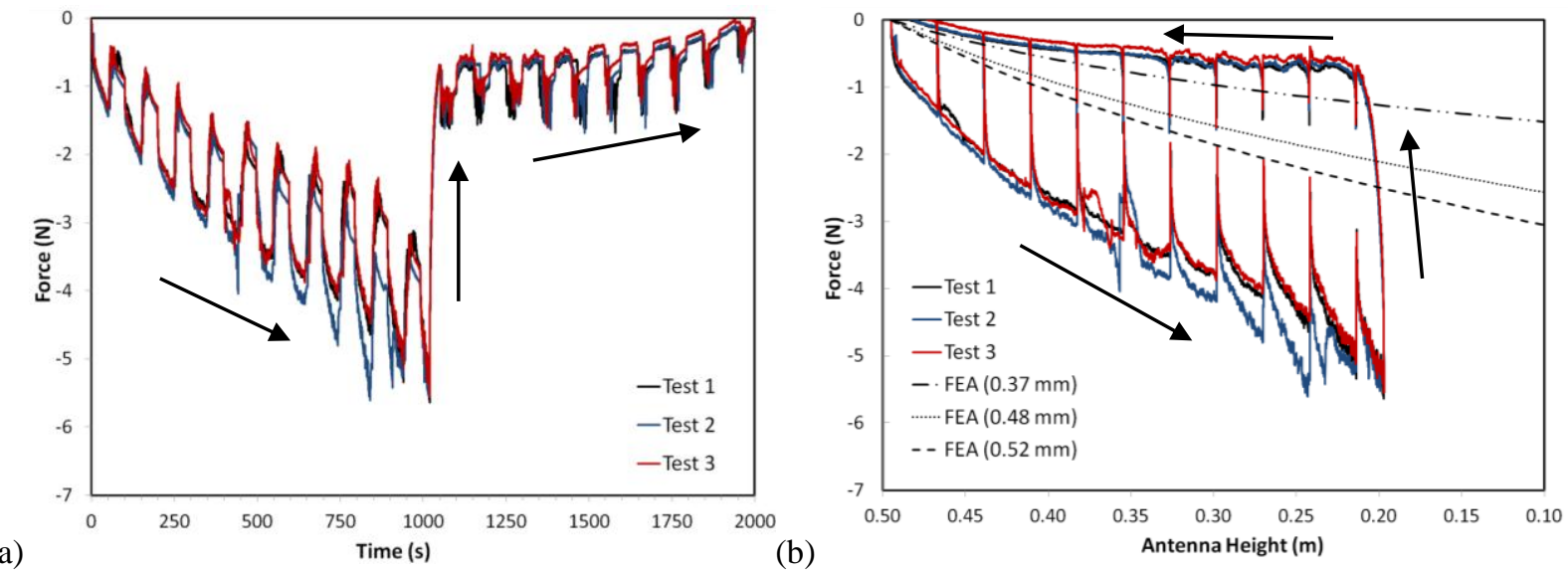

Figure 19. Compaction test friction release results plotted against (a) time and (b) height.

The compaction force results from three different finite element simulations have been overlaid on Figure 19(b). The first is for a simulation with an S-2 glass helix width of $0.367 \mathrm{~mm}$, obtained by averaging the center widths at eight different points along the four helices. The other two are results from simulations with S-2 glass widths of $0.48 \mathrm{~mm}$ and $0.52 \mathrm{~mm} ; 0.52 \mathrm{~mm}$ is the largest measured center width, while $0.48 \mathrm{~mm}$ was chosen to highlight the sensitivity of these results to helix thickness.

Considering the preliminary nature of the prototype antenna, the agreement between experiment and simulations is reasonable and it can be considered as an acceptable basis for continuing the present work and build higher quality prototypes. 


\section{B. Bending Failure Tests}

Bending failure strain tests were performed on two S-2 glass samples to determine the limit on transverse compaction for the S-2 glass helices. Each sample was initially flat and was bent and compressed in the platen test [16]. Samples were loaded and unloaded in cycles of decreasing minimum bend radius. In between cycles, the samples were removed to verify that the initially straight configuration would be recovered.

Figure 20 shows the failure load and unload cycle for the two samples. Failure of a few fibers was visually observed at a radius of curvature of 3.5 to $4 \mathrm{~mm}$ for the first sample and 4 to $4.5 \mathrm{~mm}$ for the second sample, and hence there was a noticeable difference between the load and unload cycle. Even after failure of some fibers, both samples became

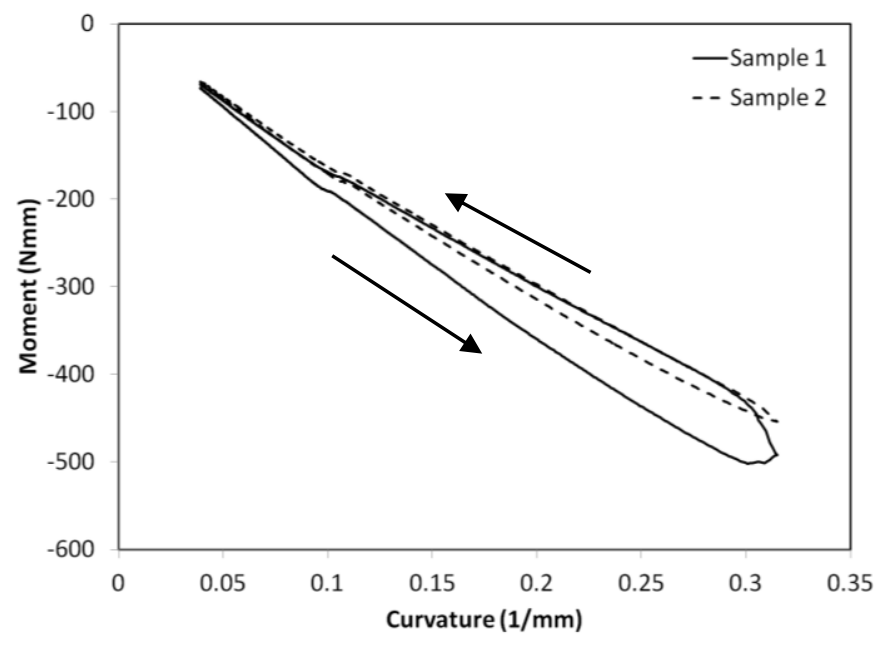

Figure 20. Bending failure test results. straight at the end of the test.

The minimum elastic bend radius for heat-treated Alloy 25 beryllium copper helices was determined from the yield stress of this material, $\sigma_{\mathrm{y}}=1350 \mathrm{MPa}$, provided in [17]. For a $0.240 \mathrm{~mm}$ thick cross-section this corresponds to a radius of curvature of $10 \mathrm{~mm}$.

Hence, the smallest allowable radius for the beryllium copper helices is larger than for the S-2 glass helices and so in our design the beryllium copper helices are the limiting factor for transverse compaction. Note that the transverse compaction results presented in Section III.C gave a maximum curvature change of $100 \mathrm{~m}^{-1}$, corresponding to a radius of $10 \mathrm{~mm}$, that is just within this limit.

\section{Reflection Coefficient Test}

A reflection coefficient test was carried out to verify the operating frequency of the prototype antenna. The results are shown below in Figure 21, and are compared to the electromagnetic simulation results.

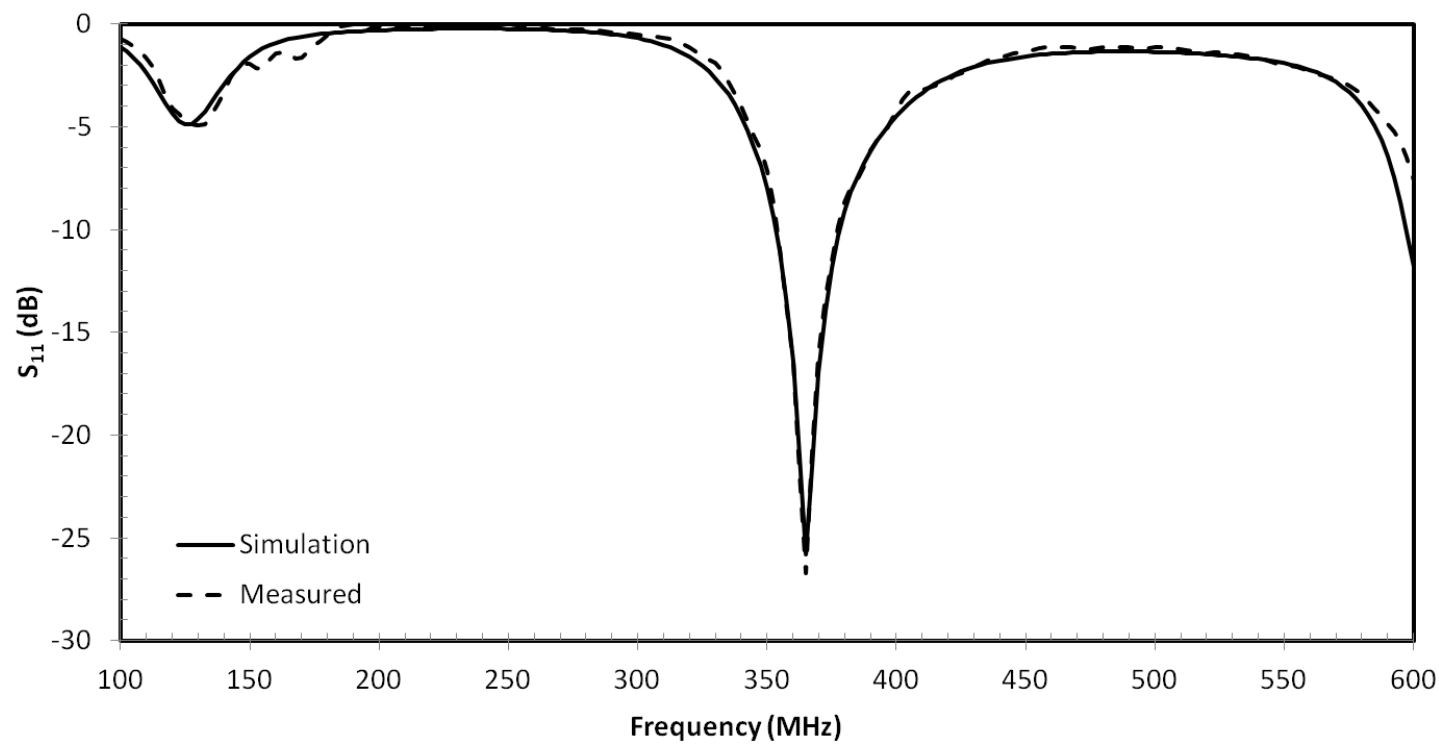

Figure 21. Prototype reflection coefficient test results.

The drop in $S_{11}$ indicates that less power is reflected back onto the network analyzer, which means this is the frequency where the antenna operates best. The results match well to the simulation, and verify that the operating 
frequency is approximately $365 \mathrm{MHz}$. Though the manufactured radius and pitch of the conductive helices varied considerably from the design dimensions, the length and cross-sectional dimensions of the conductors, which were within tolerance, are the parameters most likely to affect the operating frequency. However, the radius and pitch can both affect the gain of the antenna, which was not measured here.

\section{Conclusion}

This paper has reviewed the helical pantograph concept originally presented in [10]. Insights into the helical pantograph structure have been obtained from analytical solutions for helical rods and from finite element simulations of an eight-helix structure matching the dimensions of a physical prototype that has built and tested. Compaction force results from the simulation have been compared to compaction force experiments on the prototype; the simulation agrees with the experimental results when friction in the test set-up is removed. Concepts for transverse compaction and attachment of the structure to a CubeSat have been presented and studied using finite element simulations. Finally, the results of a reflection coefficient test on the prototype antenna have been presented, verifying the results of an electromagnetic simulation of the antenna.

The authors consider the deployable antenna concept presented here to be promising, with predictable compaction behavior, good deployed stiffness and a strong potential for self-deployment. However, further work is needed to completely characterize the structure and improve the antenna design. It is planned to build further prototypes of different dimensions and varying composite lay-ups, and to carry out further packaging and deployment studies. More antenna testing is needed to determine the gain of the antenna and how the gain is affected by an incorrect pitch or radius (which could occur through either manufacturing error or incomplete deployment). Though the antenna will operate on the correct frequency regardless of pitch or radius, the gain could be adversely affected.

Further work is needed to understand how to design the helices cross-section dimensions to optimize the structure. In this case, a linear design process was used: the cross section of the conductive helices was determined using electromagnetic simulations and then a combination of geometric and material stiffness relations was used to determine the cross section of the support helices. However, this simple approach (cross-section matching) was not effective, and a more detailed analysis is needed. Though the manufactured dimensions were considerably different from the design dimensions and the cross sections were not well matched, the structure still worked well. This suggests that there may be significant freedom in optimization of these dimensions.

In the future, the authors will move toward an integrated, iterative antenna sizing system, which considers both structural and antenna factors when selecting an optimum.

\section{Acknowledgements}

Support from the Air Force Research Laboratory Summer Space Scholars Program and AFOSR (award no. FA9550-13-1-0061, program manager Dr David Stargel) is gratefully acknowledged.

\section{References}

[1] Alhorn, D., "NanoSail-D: The Small Satellite That Could!" 25th AIAA/USU Conference on Small Satellites, Logan, UT August 2011.

[2] Glaser, D.L., "STEIN (SupraThermal Electrons, Ions and Neutrals), A New Particle Detection Instrument for Space Weather Research with CubeSats," 23rd Annual AIAA/USU Conference on Small Satellites, Logan, UT, August 2009.

[3] Patterson, K., Yamamoto, N., Pellegrino, S., "Thin Deformable Mirrors for a Reconfigurable Space Aperture," 53rd AIAA/ASME/ASCE/AHS/ASC Structures, Structural Dynamics and Materials Conference, Honolulu, HI, 23-26 April 2012.

[4] Selva, D., Kreji, D., "A survey and assessment of the capabilities of CubeSats for Earth observation," Acta Astronautica, Vol. 74, pp. 50-68, June 2012.

[5] Muri, P., Obulpathi, C., McNair, J., "Enhancing Small Satellite Communication Through Effective Antenna System Design," 2010 Military Communications Conference - Unclassified Program, San Jose, CA, November 2010.

[6] Ashida, H., "Design of Tokyo Tech Nano-Satellite Cute-1.7 + APD II and Its Operation," 59 $9^{\text {th }}$ International Astronautical Congress, Glasgow, Scotland, October 2008.

[7] Schaffner, J., "The Electronic System Design, Analysis, Integration and Construction of the Cal Poly State University CP1 CubeSat," $16^{\text {th }}$ AIAA/USU Conference on Small Satellites, Logan, UT, August 2002.

[8] King, H., Wong, J., "Characteristics of 1 to 8 wavelength uniform helical antennas," IEEE Transactions on Antennas and Propagation, Vol. 28, pp. 291-296, March 1980.

[9] Balanis, C.A., Antenna Theory: Analysis and Design, $3^{\text {rd }}$ ed, John Wiley \& Sons, 2005, Chapter 9.

[10] Olson, G., Pellegrino, S., Costantine, J., Banik, J., "Structural Architectures for a Deployable Wideband UHF Antenna," 53 AIAA/ASME/ASCE/AHS/ASC Structures, Structural Dynamics and Materials Conference, Honolulu, HI, 23-26 April 2012.

[11] You, Z., Pellegrino, S., "Cable-stiffened pantographic deployable structures. Part 1: Triangular Mast," AIAA Journal 34(4): 813-820, 
1996.

[12] "S-Band Quadrifilar Helix Antenna," <www.sstl.co.uk>, accessed March 2013.

[13] Bower, A. F., Applied Mechanics of Solids, solidmechanics.org, accessed March 2013, Chapter 10.

[14] Milton, G., "Complete characterization of the macroscopic deformations of periodic unimode metamaterials of rigid bars and pivot," Journal of Mechanics and Physics of Solids, 5 September 2012.

[15] Young, W., Budynas, R., Roark's Formulas for Stress and Strain, $7^{\text {th }}$ ed, McGraw Hill, Inc, New York, 2002 , p. 401.

[16] Sanford, G., Biskner, A., Murphey, T., "Large Strain Behavior of Thin Unidirectional Composite Flexures," 51st AIAA/ASME/ASCE/AHS/ASC Structures, Structural Dynamics and Materials Conference, Orlando, FL, 12 - 15 April 2010.

[17] "Materion: Alloy 25, Copper Beryllium Alloys,"

<http://materion.com/Products/Alloys/CopperBerylliumAlloys/Alloy25.aspx>, accessed 19 March 2013. 